\title{
Recognition Memory for Complex Visual Discriminations Is Influenced by Stimulus Interference in Rodents With Perirhinal Cortex Damage
}

\author{
Paul E. Gilbert ${ }^{1}$ and Raymond P. Kesner 2,3 \\ ${ }^{1}$ Department of Head and Neck Surgery, University of California-San Diego, San Diego, California 92103, USA; ${ }^{2}$ Department of \\ Psychology, University of Utah, Salt Lake City, Utah 84112, USA
}

\begin{abstract}
Rats with perirhinal cortex (PRC), hippocampal, or sham lesions were trained on a successive discrimination go/ no-go task to examine recognition memory for an array of visual objects with varying interference among the objects in the array. Rats were trained to recognize a target array consisting of four particular objects that could be presented in any one of four possible configurations to cover baited food wells. If the four target objects were presented, the rat should displace each object to receive food. However, if a novel object replaced any one or more of the target objects, then the rat should withhold its response. The number of novel objects presented on nonrewarded trials varied from one to four. The fewer the number of novel objects in the array, the more interference the array shared with the target array, therefore increasing task difficulty. An increased number of novel objects should result in less interference with the target array and decreased task difficulty. Although accuracy was slightly lower in rats with hippocampal lesions compared with controls, the learning of the groups was not statistically different. In contrast, rats with PRC lesions were significantly impaired in learning compared with both control and hippocampal-lesioned rats. The results suggest that recognition memory for complex visual discriminations is affected by stimulus interference in rodents with PRC damage.
\end{abstract}

Although the perirhinal cortex (PRC) was anatomically identified by Broadman (1909), its functional significance only has begun to be discovered during the past decade. In the rodent, the PRC is comprised of two strips of cortex, cytoarchitectonic areas 35 and 36, located along the banks of the rhinal sulcus (Burwell 2001). The PRC receives unimodal and polymodal input from various sensory association cortices (Burwell and Amaral 1998a). The primary efferent projection from the PRC is to entorhinal cortex (Burwell and Amaral 1998b), which provides the perforant path input into the hippocampus (Witter 1993). As described by Buffalo et al. (1998), the PRC is part of a medial temporal-lobe memory system that includes the hippocampus, dentate gyrus, subicular complex, adjacent perirhinal, entorhinal, and parahippocampal cortices.

Studies in nonhuman primates and rodents have suggested that the PRC plays a significant role in learning and memory for visual object information. Recent data suggest that PRC lesions in rodents and nonhuman primates impair visual recognition and associative memory (Meunier et al. 1993; Murray et al. 1993; Mumby and Pinel 1994; Ennaceur et al. 1996; Higuchi and Miyashita 1996; Wiig et al. 1997; Buckley and Gaffan 1998a,b; Bussey et al. 1999, 2000; Buffalo et al. 2000; Gaffan et al. 2000; Baxter and Murray 2001). In addition, neurons in the PRC and adjacent inferotemporal cortex have been shown to respond less to a visual stimulus with repeated exposure (Desimone 1996; Brown and Xiang 1998; Suzuki and Eichenbaum 2000), suggesting that PRC neurons may be involved in memory for visual objects. A large number of studies have examined the contributions of the

\section{${ }^{3}$ Corresponding author.}

E-MAIL rpkesner@behsci.utah.edu; FAX (801) 581-5841.

Article and publication are at http://www.learnmem.org/cgi/doi/10.1101/ Im.64503.
PRC and hippocampus to memory for visual objects. Data collected from nonhuman primates and rodents have shown that perirhinal lesions result in greater impairments in visual objectrecognition memory than hippocampal lesions; however, the magnitude of hippocampal impairment is a point of some contention (Meunier et al. 1993; Suzuki et al. 1993; Ennaceur et al. 1996; Zola et al. 2000). These data suggest that the PRC may be particularly involved in memory for visual objects.

A current debate exists surrounding the role of the PRC in processing visual information as described by Bussey et al. (2003). The declarative view (Buffalo et al. 1998) suggests that the PRC is involved in the mnemonic processing, but not the perceptual processing of visual stimuli. In contrast, Bussey and colleagues have proposed a perceptual-mnemonic/feature conjunction model of PRC function, which suggests that the PRC is involved in both mnemonic and perceptual processing of visual information (Bussey et al. 2002, 2003). In contrast to the declarative view, the perceptual-mnemonic/feature conjunction model suggests that impairments following PRC lesions should be related to perceptual factors, but not the speed of learning (Bussey et al. 2003). The model suggests that complex visual discriminations involving a high degree of feature ambiguity should be disrupted by PRC lesions. Feature ambiguity is described as "a property of visual discriminations that can emerge when features of an object are rewarded when they are a part of one object, but not when part of another" (Bussey et al. 2002). To test this hypothesis, Bussey et al. (2002) tested monkeys with PRC lesions on a series of concurrent discriminations consisting of a constant number of object pairs and varying degrees of feature ambiguity. In the maximum feature ambiguity condition, all features were ambiguous $(\mathrm{AB}+, \mathrm{CD}+, \mathrm{BC}-, \mathrm{AD}-)$. In the minimum feature ambiguity condition, no features were ambiguous $(\mathrm{AB}+, \mathrm{CD}+$, 
$\mathrm{EF}-, \mathrm{GH}-)$. In the intermediate condition, half of the features were ambiguous $(\mathrm{AB}+, \mathrm{CD}+, \mathrm{CE}-, \mathrm{AF}-)$. Monkeys with $\mathrm{PRC}$ lesions matched the performance of controls on discriminations involving minimum feature ambiguity. However, PRC-lesioned monkeys were mildly impaired on discriminations involving intermediate feature ambiguity, and severely impaired in the maximum feature ambiguity condition. These data suggest that the primate PRC may resolve feature ambiguity in complex visual discriminations. Therefore, a mechanism may be localized within the primate PRC that could serve to reduce interference and facilitate the resolution of stimulus ambiguity; however, this process has not been investigated in rodents.

Computational models of hippocampal function have suggested that a mechanism exists within the hippocampus for separating partially overlapping patterns, so that one pattern can be retrieved as separate from another pattern (Marr 1971; Rolls 1989, 1996; O'Reilly and McClelland 1994; Shapiro and Olton 1994). Similar to the role of the PRC in resolving feature ambiguity, this type of pattern-separation mechanism could serve to reduce interference among stimuli that share common properties. Recent behavioral studies have demonstrated that the hippocampus may be critically involved in pattern separation for spatial information (Gilbert et al. 1998; Mizumori et al. 1999; Tanila 1999). However, the role of the hippocampus in pattern separation for visual object information has not been investigated behaviorally. The present study was designed to assess the role of the rodent PRC and hippocampus in recognition memory for complex visual discriminations, using a task with varying levels of interference among stimuli in a visual array.

\section{RESULTS}

\section{Histology}

Figure 1 shows a schematic representation of a large (gray) and small (black) hippocampal lesion. Hippocampal lesions tended to produce significant bilateral cell loss within the dorsal and ventral hippocampus with minimal hippocampal savings and minimal damage to surrounding tissues, with the exception of the cortex dorsal to the hippocampus and the subiculum. An

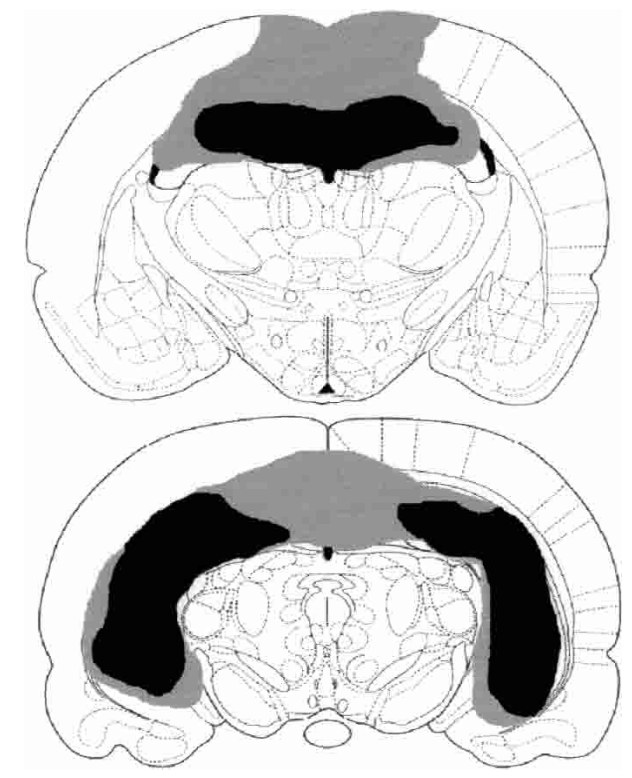

Figure 1 A schematic representation of a large (gray) and small (black) hippocampal lesion at $3.14 \mathrm{~mm}$ posterior to bregma (top) and $5.30 \mathrm{~mm}$ posterior to bregma (bottom).
Table 1. Percent Damage Following Hippocampal Lesion

\begin{tabular}{lccr}
\hline Region & $\begin{array}{c}\text { Distance Posterior } \\
\text { to Bregma (mm) }\end{array}$ & $\begin{array}{r}\text { Small } \\
\text { Lesion }\end{array}$ & $\begin{array}{r}\text { Large } \\
\text { Lesion }\end{array}$ \\
\hline Hippocampus & 3.14 & $89 \%$ & $97 \%$ \\
Subiculum & 5.30 & $66 \%$ & $89 \%$ \\
& 5.30 & $11 \%$ & $100 \%$ \\
\hline
\end{tabular}

Percent Damage Following PRC Lesion

\begin{tabular}{lcrr}
\hline Region & $\begin{array}{c}\text { Distance Posterior } \\
\text { to Bregma }(\mathrm{mm})\end{array}$ & $\begin{array}{c}\text { Small } \\
\text { Lesion }\end{array}$ & $\begin{array}{c}\text { Large } \\
\text { Lesion }\end{array}$ \\
\hline PRC & 3.14 & $66 \%$ & $94 \%$ \\
& 4.52 & $70 \%$ & $96 \%$ \\
ECT & 6.04 & $90 \%$ & $99 \%$ \\
& 3.14 & $13 \%$ & $51 \%$ \\
L Ent & 4.52 & $6 \%$ & $71 \%$ \\
& 6.04 & $8 \%$ & $67 \%$ \\
TeA & 3.14 & $26 \%$ & $43 \%$ \\
& 4.52 & $14 \%$ & $26 \%$ \\
& 6.04 & $14 \%$ & $18 \%$ \\
& 4.52 & $0 \%$ & $16 \%$ \\
& 6.04 & $0 \%$ & $19 \%$ \\
\hline
\end{tabular}

Percent damage (top) to the hippocampus and subiculum following the small and large hippocampal lesions shown in Figure 1 at 3.14 and $5.30 \mathrm{~mm}$ posterior to bregma. Percent damage (bottom) to the perirhinal cortex (PRC, area 35), ectorhinal cortex (ECT, area 36), lateral entorhinal cortex (L Ent), and temporal association cortex (TeA) following the small and large PRC lesions shown in Figure 2 at $3.14,4.52$, and $6.04 \mathrm{~mm}$ posterior to bregma.

analysis of the damage shown in Figure 1 was conducted using $\mathrm{NIH}$ Image Analysis software (http://rsb.info.nih.gov/nih-image/), and the results of the analysis are shown in Table 1. A schematic representation of a large (gray) and small (black) perirhinal cortex lesion is shown in Figure 2 (left). The schematic representation on the right shows the location of the perirhinal cortex (PRC, area 35), ectorhinal cortex (ECT, area 36), lateral entorhinal cortex (L Ent), and temporal association cortex (TeA). Lesions of the PRC produced significant bilateral damage to the PRC (area 35) and lesser damage to ectorhinal cortex (area 36). Because there is some debate regarding the inclusion of area 36 as part of PRC (for a discussion see Burwell 2001), the damage to areas 35 and 36 are reported separately in the present analysis. The lesions were intended to include both areas 35 and 36; however, there was more damage to area 35 compared with area 36 . In addition, the lesions produced some damage to lateral entorhinal cortex and temporal association cortex. An analysis of the damage shown in Figure 2 was conducted using NIH Image Analysis software, and the results of the analysis are shown in Table 1.

\section{Recognition Memory for Complex Visual Discriminations}

Figure 3 shows mean running latency (sec) as a function of novel objects in the array across four blocks of testing. The data indicate that hippocampal-lesioned rats initially showed a similar rate of learning compared with control rats on Block 1 . However, the hippocampal-lesioned rats showed a small decrease in performance on Blocks 2 and 3, but matched controls on Block 4 . Both groups displayed low latencies on target-array positive trials with " 0 " novel objects. The latencies on negative nonrewarded trials increased as a function of increased number of novel objects in the array. It is assumed that as the number of novel objects in the array increases, the ability to discriminate the array from the target array should increase. 


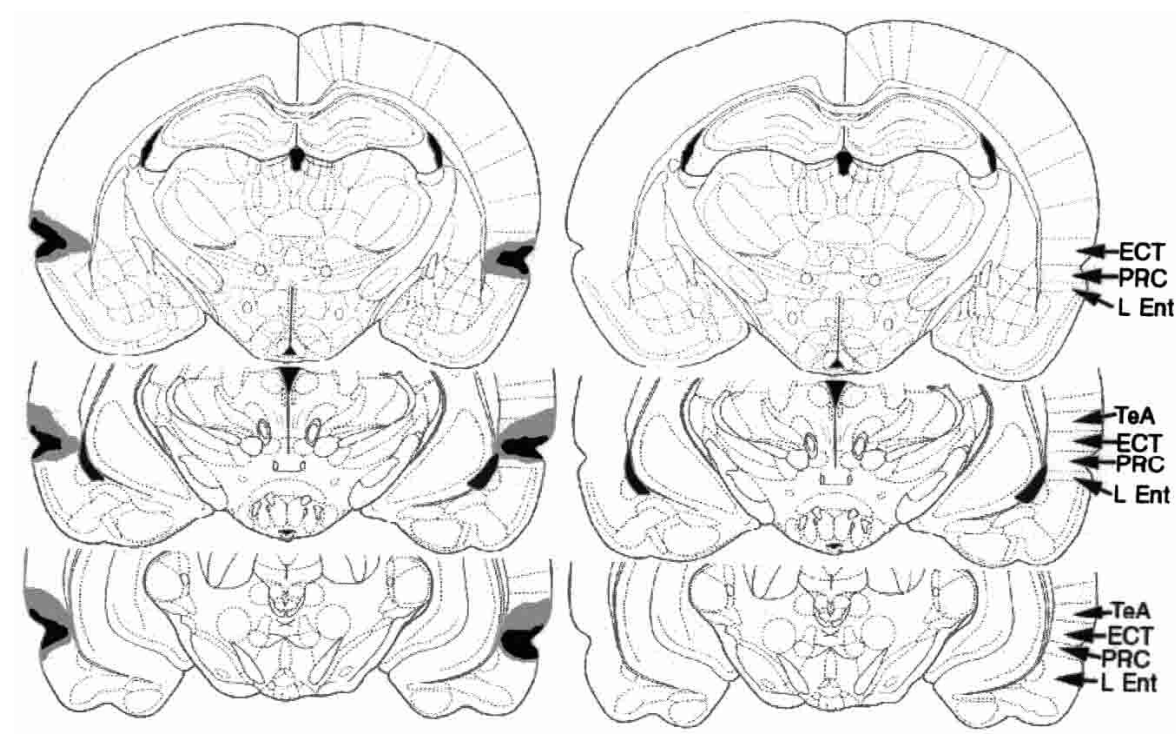

Figure 2 The schematic representation on the left shows a large (gray) and small (black) perirhinal cortex lesion at $3.14 \mathrm{~mm}$ posterior to bregma (top), $4.52 \mathrm{~mm}$ posterior to bregma (middle), and 6.04 $\mathrm{mm}$ posterior to bregma (bottom). The schematic representation on the right shows the location of the perirhinal cortex (PRC, area 35), ectorhinal cortex (ECT, area 36), lateral entorhinal cortex (L Ent), and temporal association cortex (TeA). The lesions were intended to encompass both areas 35 and 36 .

visual discriminations involving varying degrees of stimulus interference and possibly stimulus ambiguity. Rats with hippocampal lesions were slightly less accurate compared with controls; however, there were no statistically significant differences between hippocampal and control-lesioned rats, with the exception of one data point on the third block of testing. On the first block of trials, all groups showed low-latency measures on trials involving the target array, indicating that all animals were displacing the target object on rewarded trials. On trials involving one novel object (maximum interference condition), all groups showed low-latency measures, suggesting that even control rats had a difficult time performing the task when interference and task difficulty were high. However, on trials involving two, three, or four novel objects (decreasing interference), control and hippocampal-lesioned rats showed longer latencies, suggesting that the rats were able to discriminate the novel arrays from the target array and withhold their

Lesions of the PRC resulted in deficits across all blocks of testing relative to controls and hippocampal-lesioned rats. However, the PRC-lesioned group showed some improvement across blocks and was not significantly different from controls on nonrewarded trials with four novel objects involving no stimulus interference.

The data were grouped into four blocks of 40 trials for analysis. Each block represents $1 \mathrm{wk}$ of five consecutive days of testing. Each day- 4 target arrays was presented along with four arrays containing novel objects. A repeated-measures three-way analysis of variance with lesion (control, hippocampus, PRC) as the between factor and block $(1,2,3,4)$ and novel objects $(0,1,2,3$, $4)$ as the within factors, revealed a significant main effect of lesion, $\mathrm{F}(2,12)=8.68, P<0.01$, a significant main effect of novel objects $\mathrm{F}(4,48)=480.69, P<0.0001$, a significant novel objects $\times$ lesion interaction $\mathrm{F}(8,48)=15.63, P<0.0001$, a significant block $\times$ novel objects interaction $\mathrm{F}(12,144)=15.28, P<0.0001$, and a significant lesion $\times$ block $\times$ novel objects interaction $F(24$, $144)=2.28, P<0.01$. A Tukey's HSD test for multiple comparisons on the main effect of a group revealed that there were no statistically significant differences $(P<0.05)$ between the hippocampal-lesioned rats and the control group. However, the test revealed statistically significant differences $(P<0.05)$ between the rats with PRC lesion and both the controls and hippocampallesioned rats. Because the analysis revealed a significant lesion $\times$ block $\times$ novel objects interaction, a two-way lesion $\times$ novel objects multivariate analysis of variance (MANOVA) was conducted on each block, and Tukey's HSD tests for multiple comparisons were used to examine differences among the three groups. For the condition in which there were three novel objects, the perirhinal cortex group displayed a significant reduction in mean latency relative to controls and hippocampal-lesioned rats for Blocks 1,2 , and $4(P<0.01)$, but for Block 3 , both the hippocampus and PRC-lesioned group displayed a reduced latency relative to controls $(P<0.0001)$.

\section{DISCUSSION}

The present data demonstrate that rats with PRC lesions were significantly impaired relative to controls in learning-complex response. In contrast, rats with PRC lesions continued to show low latencies on trials involving two and three novel objects, indicating that the rats were unable to discriminate arrays involving novel objects from the target array even when stimulus interference was minimal. Because the PRC-lesioned rats were not significantly different from control and hippocampal-lesioned rats on trials with four novel objects (no stimulus interference), the data suggest that PRC-lesioned rats are able to discriminate between the target array and an array with no similarity. This finding demonstrates that PRC lesions do not completely abolish visual discrimination, and that rats with PRC lesions are able to perform visual discriminations when there is no stimulus interference.

On the second block of testing, the performance of each group was similar to the first block of testing. The hippocampallesioned rats showed a decrease in performance relative to controls; however, the difference was not statistically significant. The PRC-lesioned rats showed some improvement on trials involving two novel objects (medium interference) and four novel objects (no interference), but still showed significant impairment relative to controls. On the third block of trials, control rats continued to outperform PRC-lesioned rats on trials with three novel objects. There was also a significant difference between the control rats and rats with hippocampal lesions on trials with three novel objects. However, by the fourth block of trials, the hippocampal-lesioned group was indistinguishable from controls, but the rats with PRC lesions continued to show a deficit relative to both the controls and hippocampal-lesioned rats. These data suggest that rats with PRC lesions are unable to perform complex visual discriminations involving interference among arrays of visual stimuli. Therefore, a mechanism may be localized within the rodent PRC that could serve to reduce interference and facilitate recognition memory for complex visual discriminations, particularly when task difficulty and interference are increased.

The deficits in visual memory associated with PRC lesions in the present study are consistent with previous data collected from rodents and nonhuman primates (Meunier et al. 1993; Murray et al. 1993; Mumby and Pinel 1994; Ennaceur et al. 1996; Higuchi and Miyashita 1996; Wiig et al. 1997; Buckley and 


\section{BLOCK 1}

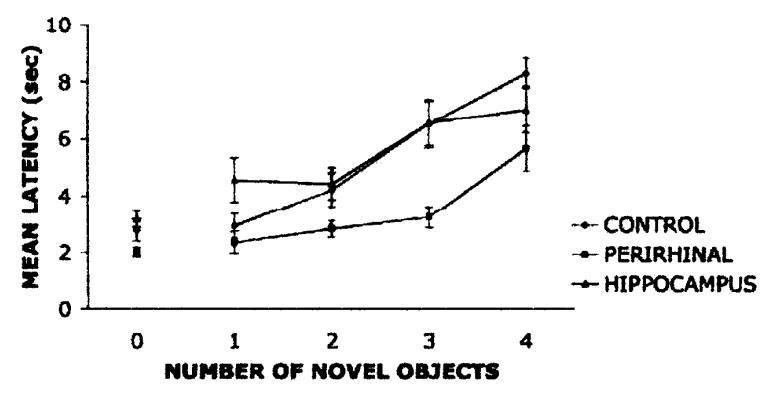

BLOCK 2

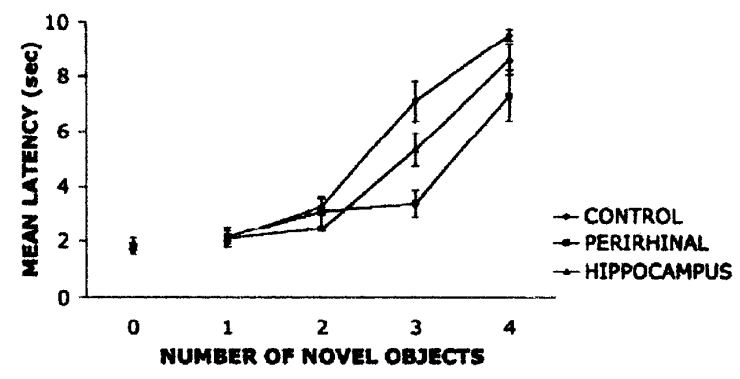

BLOCK 3

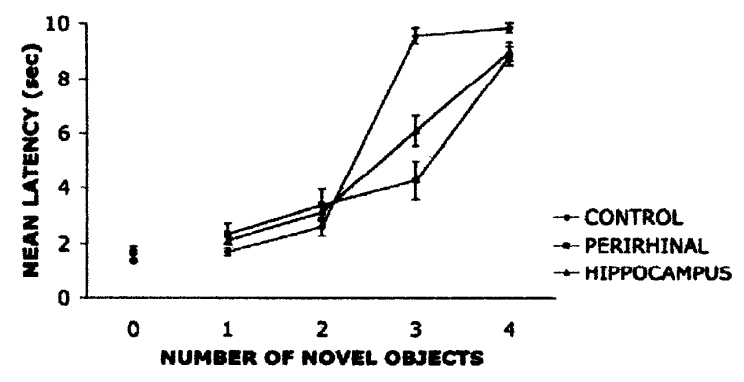

BLOCK 4

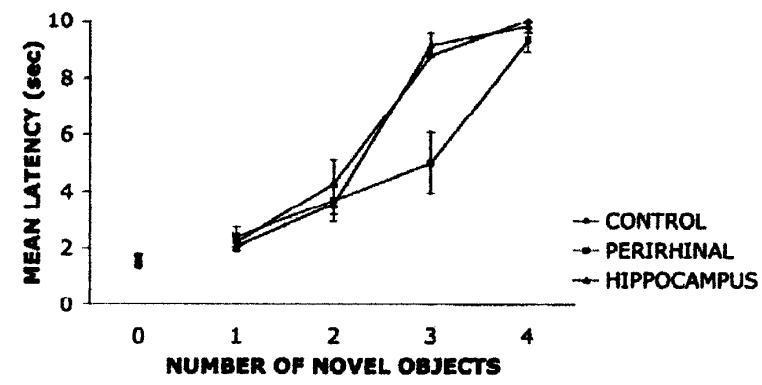

Figure 3 Mean latency scores as a function of the number of novel objects in an array of visual objects from sham (control), hippocampal, and perirhinal cortex-lesioned rats on four blocks of trials. Trials with zero novel objects were rewarded target trials, whereas trials containing one or more novel objects were nonrewarded trials. It is hypothesized that as the number of novel objects in the array increases, the ability to discriminate the array from the target array should increase.
Gaffan 1998a,b; Bussey et al. 1999, 2000; Buffalo et al. 2000; Gaffan et al. 2000; Baxter and Murray 2001). In particular, a prior study in monkeys demonstrated that the primate PRC might resolve feature ambiguity in complex visual discriminations (Bussey et al. 2002). The results of the present study could be interpreted to suggest that the rodent PRC also may resolve feature ambiguity in visual discrimination tasks; however, this interpretation is confounded by differences in task difficulty. Therefore, further studies should be conducted to examine the role of the rodent PRC in resolving feature ambiguity. However, the results of the present experiment offer further support for the proposal that the PRC may serve similar functions in rodents and primates (Burwell et al. 1995; Gaffan et al. 2000).

The lack of a significant deficit in rats with hippocampal lesions suggests that the hippocampus is not involved in reducing interference for visual discriminations. As mentioned previously, computational models of hippocampal function have suggested that a pattern separation mechanism exists within the hippocampus that could serve to reduce interference among stimuli (Marr 1971; Rolls 1989, 1996; O'Reilly and McClelland 1994; Shapiro and Olton 1994). Prior behavioral studies have demonstrated that the hippocampus may be critically involved in pattern separation for spatial information (Gilbert et al. 1998; Mizumori et al. 1999; Tanila 1999). However, assuming that the present task requires pattern separation for visual objects, the results suggest that the hippocampus does not play a significant role in pattern separation for visual object information. Four different configurations were used in the present study to minimize any spatial component of the task. The use of different configurations forced the rats to attend to each of the individual visual objects in the array and minimized any association between an object and a particular spatial location. It was important to minimize the spatial component of the task, because prior studies have shown that PRC lesions impair object-place conditional learning in rats (Bussey et al. 2001; Liu and Bilkey 2001). If the present task involved objects associated with particular locations, the interpretation of the data may have been confounded by the object-place associative learning component of the task. Although the location of each object was not relevant to task performance, a spatial relationship did exist among the four objects in the array. This spatial relationship among the objects may have resulted in the slight deficit observed in the hippocampallesioned rats on the present task. However, if the task performance involved a spatial component, it is hypothesized that hippocampal lesions would have resulted in a learning deficit.

The results of the present study suggest that the rodent PRC is involved in recognition memory for complex visual discriminations, particularly when interference among visual objects is increased. However, the data suggest that the hippocampus is not necessary for accurate visual object-recognition memory. The present results support the proposal that the PRC may serve similar functions in rodents and primates (Burwell et al. 1995; Gaffan et al. 2000), and the data offer support for the perceptual-mnemonic/feature conjunction model (Bussey et al. 2003).

\section{MATERIALS AND METHODS}

\section{Subjects}

Fifteen male Long-Evans rats, $\sim 2$ mo of age and weighing $\sim 350 \mathrm{~g}$ at the start of the experiment, were used as subjects. Each rat was housed individually in metal hanging cages located in a colony room. The colony room was maintained on a $12 \mathrm{~h}$ light/12 $\mathrm{h}$ dark cycle. All rats had unlimited access to water, but were initially food deprived to $85 \%-90 \%$ of free feeding weight. All testing was conducted during the light portion of the light/dark cycle. 


\section{Apparatus}

The testing apparatus consisted of a box with an $84-\mathrm{cm} \times 27-\mathrm{cm}$ painted wood floor, and four $30.5-\mathrm{cm}$ high nontransparent red Plexiglas walls (see Fig. 4). One removable red Plexiglas guillotine door was placed $25 \mathrm{~cm}$ from one end of the box to divide the box into two separate compartments. The small $25-\mathrm{cm}$ long compartment served as the start chamber, in which the rat began each trial, and the larger $59-\mathrm{cm}$ long compartment served as the choice chamber in which the objects were presented. A $5 \times 4$ matrix of evenly spaced $2-\mathrm{cm}$ diameter and $1.5-\mathrm{cm}$ deep food wells were drilled in the floor at both ends of the apparatus. The rows and columns of food wells were each separated by $2.5 \mathrm{~cm}$.

\section{Preoperative Shaping Procedure}

During the first week of training, each animal was handled for $\sim 0.25 \mathrm{~h}$ daily and was allowed to individually explore the test apparatus for $0.25 \mathrm{~h}$. During the exploration period, $\sim 10$ pieces of Froot Loop cereal were spread out across the surface of the apparatus in the choice chamber. Beginning the second week of training, a single, neutral object was introduced to the testing apparatus. The object was used to shape each animal to displace an object to receive a food reward. The object consisted of a wooden object $2-\mathrm{cm}$ wide and $5-\mathrm{cm}$ tall that was painted gray. The object was placed to cover the centermost food-well in the choice chamber of the apparatus. On each shaping trial, a piece of cereal was placed in front of the object on the maze surface. The animal was placed in the start chamber of the apparatus with the guillotine door between the start and choice chambers in the closed position. The animal was allowed to exit the start chamber, retrieve the reward from the choice chamber, and return to the start chamber to consume the reward with the door in the closed position. This procedure was followed 12 times each day. Once an animal retrieved the food reward consistently, the food reward was placed in the food well previously covered by the object, and the object was positioned on the side of the food well opposite the animal. On each ensuing trial, the object was positioned to cover a larger portion of the food well, until the base of the object covered the baited food well completely. Once an animal consistently displaced the object to receive a food reward, the animal was scheduled for surgery.
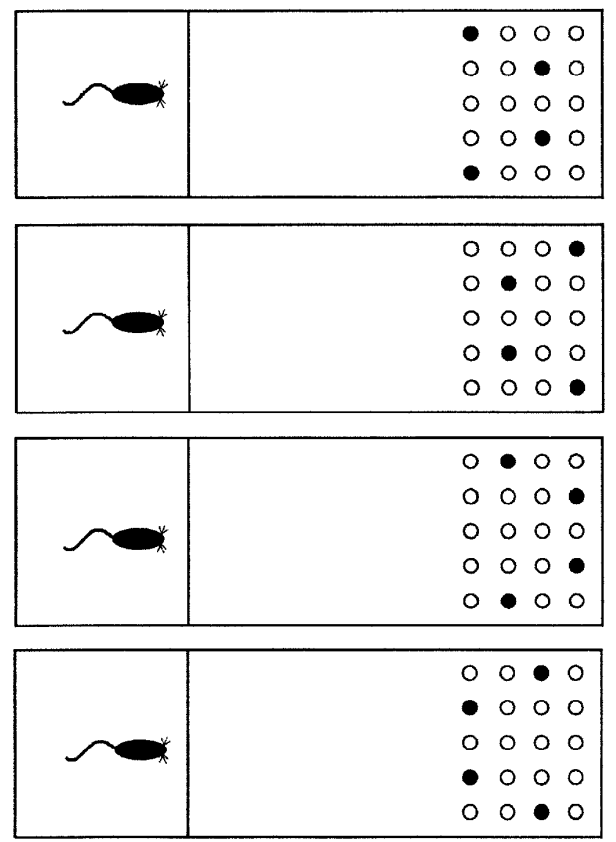

Figure 4 Schematics of the apparatus and the four array configurations indicated by the black-filled circles.

\section{Surgery}

Each animal was assigned randomly to receive a bilateral electrolytic-induced lesion of the hippocampus $(n=5)$, a bilateral aspiration lesion of the perirhinal cortex, or a sham lesion $(n=5)$ prior to testing on the task. Prior to surgery, each animal was given atropine sulfate $(0.25 \mathrm{mg} / \mathrm{kg}$, i.p. $)$ and anesthetized with sodium pentobarbital (Nembutal; $60 \mathrm{mg} / \mathrm{mL}$, i.p.). Each animal was then placed in a stereotaxic instrument and an incision was made in the skin covering the skull. For rats in the hippocampallesioned group, the bone overlying the hippocampus was removed with a small dental burr. Electrolytic lesions were generated by passing a 1.2-mA anodal current for $10 \mathrm{sec}$ through a stainless-steel electrode $(0.35 \mathrm{~mm}$ in diameter $)$ insulated with Epoxylite, except for $\sim 0.50-0.75 \mathrm{~mm}$ at the tip of the electrode. The coordinates for the electrolytic-induced hippocampal lesions were $3.5 \mathrm{~mm}$ posterior to bregma; $1.0,2.2$, and $3.4 \mathrm{~mm}$ lateral to midline; and $2.8 \mathrm{~mm}$ below dura for dorsal hippocampus. For the ventral hippocampus, the coordinates were $4.6 \mathrm{~mm}$ posterior to bregma, $5.5 \mathrm{~mm}$ lateral to midline, and 5.6 and $8.1 \mathrm{~mm}$ ventral to dura. For rats in the perirhinal lesion group, the temporal muscle was retracted to reveal the skull overlying the rhinal fissure. The bone overlying the rhinal fissure from $3.0 \mathrm{~mm}$ to 7.0 $\mathrm{mm}$ posterior to bregma was removed. A small incision was made in the dura mater to allow aspiration of the perirhinal cortex. The perirhinal cortex dorsal and ventral to the rhinal fissure was then removed with an aspiration needle under microscopic visual guidance. Following aspiration, the temporal muscle was returned to its position. Rats in the sham lesion condition received the same surgical treatment; however, no bone was removed. Following all surgical procedures, each animal was sutured, injected with $1.5 \mathrm{cc}$ (sc) saline in each hip, and placed on a heating pad to recover. The subcutaneous injections of saline were to rehydrate the animal to avoid dehydration following surgery and promote the excretion of the sodium pentobarbital through urination.

\section{Task Procedure}

Following a 7-10 d recovery period from surgery, each rat was trained on a successive discrimination go/no-go task to examine pattern separation for visual objects. Each rat was given eight trials per day. Rats were trained to recognize a target array consisting of four particular, very different objects. Each object consisted of a small toy (4-10 cm high) mounted on a small flat metal washer (5 $\mathrm{cm}$ in diameter). These four objects could be presented in any one of four possible array configurations (Fig. 4 ), and each object could occur in any one of the four positions within the configuration, thus minimizing the spatial component and requiring the animals to attend to each object on each trial. The four target objects covered four baited food-wells. Thus, if the four target objects were presented on any given trial, in any one of the four configurations, the rat should learn to run and displace the objects to receive food rewards (go). However, if a novel object replaced any one or more of the objects in the array, then the rat should withhold its response and not displace the objects (no-go). The number of novel objects in the no-go condition varied on each trial, from one to four objects. The novel objects consisted of 50 toys $(4-10 \mathrm{~cm}$ high) mounted on metal washers (5 $\mathrm{cm}$ in diameter). Each novel object was only presented once during each block (week) of testing. The latency of each rat to displace the first object of the set was measured, recorded by the experimenter, and used as the dependent measure. If the rat did not displace an object within $10 \mathrm{sec}$, a latency of $10 \mathrm{sec}$ was recorded, and the rat was returned to the start chamber to await the next trial. The fewer the number of novel objects in the array, the more similarity the array shared with the target array, therefore increasing interference and task difficulty. However, an increased number of novel objects in the array should result in less similarity with the target array and decreased task difficulty. It is suggested that the nonrewarded trials with increased similarity to the target array should require more reduction of interference than trials with less similarity. Each day, four positive go target arrays and four negative no-go arrays were presented. Of the four 
negative no-go arrays presented each day, there was one array with one, two, three, and four novels objects presented. Each rat was tested on the task for two blocks of 160 trials. Each block consisted of 80 positive go trials and 80 negative no-go trials. Of the 80 negative no-go trials, there were 20 trials consisting of one, two, three, or four novel objects.

\section{Histology}

At the conclusion of all testing, each animal was deeply anesthetized with an intraperitoneal injection of $1 \mathrm{~mL}$ sodium pentobarbital $(100 \mathrm{mg} / \mathrm{kg})$ and perfused intracardially, followed by a $10 \%$ formalin solution. The brain was removed from the skull and stored in a $10 \%$ formalin/30\% sucrose solution. For animals with either complete hippocampal or control lesions, each brain was frozen and cut at $24-\mu \mathrm{m}$ sections, starting at bregma and extending through the posterior region of either the hippocampus or perirhinal cortex. Every third section was mounted on a glass slide, stained with cresyl violet, and examined for histological verification of the lesion placement.

\section{ACKNOWLEDGMENTS}

Research was supported by NSF Grant IBN 9817583 awarded to R.P.K., and NIH NRSA Fellowship MH 12742-01 awarded to P.E.G. We thank Russell Millham, Amra Peterson, Lindsay Barua, Creed Stobbe, Connie Linkenhoker, and Kena Hansen for their assistance in data collection. In addition, we thank Benjamin Loveridge for his assistance with surgical procedures and Benjamin Kuntz for his capable histological work.

The publication costs of this article were defrayed in part by payment of page charges. This article must therefore be hereby marked "advertisement" in accordance with 18 USC section 1734 solely to indicate this fact.

\section{REFERENCES}

Baxter, M.G. and Murray, E.A. 2001. Impairments in visual discrimination learning and recognition memory produced by neurotoxic lesions of rhinal cortex in rhesus monkeys. Eur. J. Neurosci. 13: 1228-1238.

Broadman, K. 1909. Vergleichende Lokalisationslehre der Grosshirnrinde. Barth, Leipzig, Germany. [Title translation: Comparative study of localization in the cerebral cortex.]

Brown, M.W. and Xiang, J.Z. 1998. Recognition memory: Neuronal substrates of the judgment of prior occurrence. Prog. Neurobiol. 55: $149-189$.

Buckley, M.J. and Gaffan, D. 1998a. Perirhinal cortex ablation impairs visual object identification. J. Neuorsci. 18: 2268-2275

- 1998b. Perirhinal cortex ablation impairs configural learning and paired-associate learning equally. Neuropsychologia 36: 535-546.

Buffalo, E.A., Reber, P.J., and Squire, L.R. 1998. The human perirhinal cortex and recognition memory. Hippocampus 8: 330-339.

Buffalo, E.A., Ramus, S.J., Clark, R.E., Teng, E., Squire, L.R., and Zola-Morgan, S.M. 2000. Dissociation between the effects of damage to perirhinal cortex and area TE. Learn. Mem. 7: 375-382.

Burwell, R.D. 2001. Borders and cytoarchitecture of the perirhinal and postrhinal cortices in the rat. J. Comp. Neurol. 437: 17-41.

Burwell, R.D. and Amaral, D.G. 1998a. Cortical afferents of the perirhinal and postrhinal, and entorhinal cortices of the rat. J. Comp. Neurol. 398: 179-205.

- 1998b. Perirhinal and postrhinal cortices of the rat: Interconnectivity and connections with the entorhinal cortex. $J$. Comp. Neurol. 391: 293-321.

Burwell, R.D., Witter, M.P., and Amaral, D.G. 1995. Perirhinal and postrhinal cortices of the rat: A review of the neuroanatomical literature and comparison with findings from the monkey brain. Hippocampus 5: 390-408.

Bussey, T.J., Muir, J.L., and Aggelton, J.P. 1999. Functionally dissociating aspects of event memory: The effects of combined perirhinal and postrhinal cortex lesions on object and place memory in the rat. $J$. Neurosci. 19: 495-502.

Bussey, T.J., Duck, J., Muir, J.L., and Aggelton, J.P. 2000. Distinct patterns of behavioural impairments resulting from fornix transection or neurotoxic lesions of the perirhinal and postrhinal cortices in the rat. Behav. Brain Res. 111: 187-202.

Bussey, T.J., Dias, R., Amin, E., Muir, J.L., and Aggelton, J.P. 2001. Perirhinal cortex and place-object conditional learning in the rat. Behav. Neurosci. 115: 776-785.

Bussey, T.J., Saksida, L.M., and Murray, E.A. 2002. Perirhinal cortex resolves feature ambiguity in complex visual discriminations. Eur. J. Neurosci. 15: 365-374.

Bussey, T.J., Saksida, L.M., and Murray, E.A. 2003. Impairments in visual discrimination after perirhinal cortex lesions: Testing 'declarative' vs. 'perceptual-mnemonic' views of perirhinal cortex function. Eur. J. Neurosci. 17: 649-660.

Desimone, R. 1996. Neural mechanisms for visual memory and their role in attention. Proc. Natl. Acad. Sci. 93: 13494-13499.

Ennaceur, A., Neave, N., and Aggelton, J.P. 1996. Neurotoxic lesions of the perirhinal cortex do not mimic the behavioral effects of fornix transection in the rat. Behav. Brain Res. 80: 9-25.

Gaffan, E., Eacott, M., and Simpson, E. 2000. Perirhinal cortex ablation in rats selectively impairs object identification in a simultaneous visual comparison task in rats. Behav. Neurosci. 114: 18-31.

Gilbert, P.E., Kesner, R.P., and DeCoteau, W.E. 1998. The role of the hippocampus in mediating spatial pattern separation. J. Neurosci. 18: $804-810$.

Higuchi, S. and Miyashita, Y. 1996. Formation of mnemonic neuronal responses to visual paired associates in inferotemporal cortex is impaired by perirhinal and entorhinal lesions. Proc. Natl. Acad. Sci. 93: 739-743.

Liu, P. and Bilkey, D.K. 2001. The effect of excitotoxic lesions centered on the hippocampus or perirhinal cortex in object recognition and spatial memory tasks. Behav. Neurosci. 115: $94-111$.

Marr, D. 1971. Simple memory: A theory for archicortex. Philos. Trans. R. Soc. Lond. B Biol. Sci. 262: 23-81.

Meunier, M., Bachevalier, J., Mishkin, M., and Murray, E.A. 1993. Effects on visual recognition of combined and separate ablations of the entorhinal and perirhinal cortex in rhesus monkeys. J. Neurosci. 13: 5418-5432.

Mizumori, S.J.Y., Ragozzino, K.E., Cooper, B.G., and Leutgeb, S. 1999. Hippocampal representational organization and spatial context. Hippocampus 9: 444-451.

Mumby, D.G. and Pinel, J.P.J. 1994. Rhinal cortex lesions and object recognition in rats. Behav. Neurosci. 108: 11-18.

Murray, E.A., Gaffan, D., and Mishkin, M. 1993. Neural substrates of visual stimulus-stimulus association in rhesus monkeys. J. Neurosci. 13: $4549-4561$.

O'Reilly, R.C. and McClelland, J.L. 1994. Hippocampal conjunctive encoding, storage, and recall: Avoiding a trade-off. Hippocampus 4: 661-682.

Rolls, E.T. 1989. Functions of neuronal networks in the hippocampus and neocortex in memory. In Neural models of plasticity: Theoretical and empirical approaches (eds. J.H. Byrne and W.O. Berry), pp. 240-265. Academic Press, New York.

. 1996. A theory of hippocampal function in memory. Hippocampus 6: 601-620.

Shapiro, M.L. and Olton, D.S. 1994. Hippocampal function and interference. In Memory systems 1994. (eds. D.L. Schacter and E. Tulving), pp. 141-146. MIT Press, London.

Suzuki, W.A. and Eichenbaum, H. 2000. The neurophysiology of memory. Ann. NY Acad. Sci. 911: 175-191.

Suzuki, W.A., Zola-Morgan, S., Squire, L.R., and Amaral, D.G. 1993 Lesions of the perirhinal and parahippocampal cortices in the monkey produce long-lasting memory impairment in the visual and tactual modalities. J. Neurosci. 13: 2430-2451.

Tanila, H. 1999. Hippocampal place cells can develop distinct representations of two visually identical environments. Hippocampus 9: 235-246.

Wiig, K.A., Bear, M.F., and Burwell, R.D. 1997. Comparable memory impairment following electrolytic or neurotoxic lesion of the rat perirhinal cortex. Soc. Neurosci. Abst. 23: 1599.

Witter, M.P. 1993. Organization of the entorhinal-hippocampal system: A review of current anatomical data. Hippocampus 3: 33-44.

Zola, S.M., Squire, L.R., Teng, E., Stefanacci, L., Buffalo, E.A., and Clark, R.E. 2000. Impaired recognition memory in monkeys after damage limited to the hippocampal region. J. Neurosci. 20: 451-463.

Received June 18, 2003; accepted in revised form September 22, 2003. 


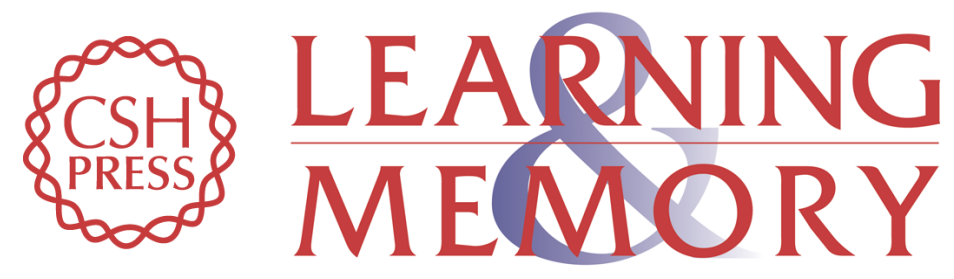

\section{Recognition Memory for Complex Visual Discriminations Is Influenced by Stimulus Interference in Rodents With Perirhinal Cortex Damage}

Paul E. Gilbert and Raymond P. Kesner

Learn. Mem. 2003, 10:

Access the most recent version at doi:10.1101//m.64503

References This article cites 33 articles, 10 of which can be accessed free at: http://learnmem.cshlp.org/content/10/6/525.full.html\#ref-list-1

License

Email Alerting Receive free email alerts when new articles cite this article - sign up in the box at the Service top right corner of the article or click here. 